OPEN ACCESS

Edited by:

Giorgio Mattana,

Université de Paris, France

Reviewed by:

Niravkumar J. Joshi,

University of Sao Paulo, Brazil

M. ljaz Khan,

Peking University, China

*Correspondence: Ismael Domingos

ismael.domingos@ua.pt

Helena Alves

alves.helena@ua.pt

Specialty section:

This article was submitted to Interdisciplinary Physics,

a section of the journal

Frontiers in Physics

Received: 16 July 2021 Accepted: 27 September 2021

Published: 18 October 2021

Citation:

Domingos I, Neves AIS, Craciun MF and Alves H (2021) Graphene Based

Triboelectric Nanogenerators Using

Water Based Solution Process.

Front. Phys. 9:742563.

doi: 10.3389/fphy.2021.742563

\section{Graphene Based Triboelectric Nanogenerators Using Water Based Solution Process}

\author{
Ismael Domingos ${ }^{1 *}$, Ana I. S. Neves ${ }^{2}$, Monica F. Craciun ${ }^{2}$ and Helena Alves ${ }^{1 *}$ \\ ${ }^{1}$ CICECO, Physics and Chemistry Department, University of Aveiro, Aveiro, Portugal, ${ }^{2}$ Centre for Graphene Science, College of \\ Engineering, Mathematics and Physical Sciences, University of Exeter, Exeter, United Kingdom
}

A rapid development in personal electronics has raised challenging requirements for portable and sustainable power sources. For example, in wearable technologies, the concept of wearable body area network brings body motion and vital signs monitoring together in synergy. For this, a key aspect is sustainable portable energy, available anywhere, at any time, as generated by triboelectric nanogenerators (TENG). This technology usually demands high-cost processes and materials and still suffer from low power output, as well as unstable output values due to charge generating stimulus with variable intensities. In this work, we present TENGs using shear exfoliated graphene as electrodes as well as active triboelectric layer deposited by a simple solution process. Graphene in combination with polymers such as polydimethylsiloxane (PDMS) were used to produce TENG devices using low-cost solution processing methods. Device electrical power generation was tested with a cyclic physical stimulus for better control and understanding of device output. The triboelectric response of these materials showed open circuit voltages $\left(V_{o c}\right)$ and shortcircuit currents $\left(\mathrm{I}_{\mathrm{sc}}\right)$ of approximately $233 \mathrm{~V}$ and $731 \mathrm{nA}$ respectively when stimulated at $1.5 \mathrm{~Hz}$. A power density of $13.14 \mu \mathrm{W} / \mathrm{cm}^{2}$ under a load of $200 \mathrm{M} \Omega$ was achieved, which can be 40 times higher when compared to devices made with aluminum and PDMS. These results demonstrate the potential of solution process for low-cost triboelectric devices for self-sustainable wearable portable nanogenerators on health and security applications using contact and positional sensors.

\footnotetext{
Keywords: nanogenerator, energy harvesting, triboelectric nanogenerators, graphene, loT, wearable body area network, triboelectric generator
}

\section{INTRODUCTION}

Among sustainable energy-harvesting sources [1], mechanical energy is one of the most versatile and commonly available, capable to contribute to milliwatts and sometimes even few watts of energy produced by human body motion $[2,3]$. This energy, if efficiently harvested through triboelectric nanogenerators (TENG), could be sufficient for autonomous operation of small IoT, wearable or medical devices, sensors and actuators [2-5]. These can be safer since their output currents are usually in microampere level, using low-cost, lightweight, and adaptable designs as well as able to provide high voltages owing to their ultralow capacitance [6]. 2D materials, as graphene, have emerged as effective materials for TENGs, either as active triboelectric layers or electrodes with tuneable work function [7-10], mechanical conformability [11], and sensitivity [12]. Unfortunately, 
current techniques and processes used for device fabrication use complex growth processes based on physical and chemical vapor deposition, which are difficult to scale up [13, 14]. To allow simpler fabrication and low costs, there is a need to implement solution-based processes, preferentially with low environmental impact solvents such as water.

Here we present a graphene based triboelectric nanogenerator were this material acts both as electrode and as active triboelectric layer. All graphene layers were developed using shear exfoliated graphene and transferred using vacuum filtration techniques to integrate easy and low-cost solution-based methods. This device takes advantage of the triboelectric effect between PDMS and graphene and was developed with an extra polymeric layer to prevent current leakage from the PDMS layer to its back electrode. The output power behaviour associated with the external load connected to the TENG is similar to a device fabricated using a standard PDMS and aluminum triboelectric pair.

\section{MATERIALS AND METHODS}

\section{Materials}

Graphite flakes and sodium cholate (97\%) were purchased from Sigma-Aldrich. PDMS solution- Sylgard 184 was acquired from Farnell element14 Components, S.L. Polytetrafluoroethylene (PTFE) filter membranes with $0.2 \mu \mathrm{m}$ pore and $47 \mathrm{~mm}$ diameter were from Omnipore. Polyimide (Kapton) Film Electrical Tape 1205 was from 3M. Aluminum (Al) foil was of conventional domestic use.

\section{Methods}

Shear Exfoliation of Graphite: Shear-exfoliated graphene (SEG) was produced by shear exfoliation of graphite powder in deionized water with sodium cholate as surfactant similarly to previously reported work [15]. The exfoliation was carried out using a high shear laboratory mixer (L5M, Silverson Machines Ltd.) with a $32 \mathrm{~mm}$ diameter rotor head and rectangular perforations. $15 \mathrm{mg} / \mathrm{ml}$ of graphite flakes and $5 \mathrm{mg} / \mathrm{ml}$ of surfactant where mixed at 4,500 rpm for $60 \mathrm{~min}$. The resulting suspension was left to rest overnight. Then a decanting step was performed to remove the unexfoliated excess graphite followed by centrifugation at $1,500 \mathrm{rpm}$ for $60 \mathrm{~min}$ for further separation.

SEG/TENG fabrication: $2.5 \times 2.5 \mathrm{~cm}$ glass substrates were submitted to a cleaning process and surface treatment prior to SEG deposition. A combination of three $15 \mathrm{~min}$ baths of distilled water, acetone and isopropanol respectively was used for cleaning. Corona discharge surface treatment was performed along all the sample surface with a high frequency generator from Electro-Technic products model BD-20 at a distance of $1 \mathrm{~cm}$ for $10 \mathrm{~s}$. Glass substrates were coated with graphene films from SEG suspensions using an isopropyl alcohol-assisted direct transfer method (IDT). This solution based transferring technique consists of filtrating the SEG suspensions through a membrane and then transferring the resulting film from the membrane to the substrate through isopropyl alcohol (IPA) evaporation. PTFE membranes with $4.7 \mathrm{~cm}$ of diameter were used for vacuum filtration of $10 \mathrm{ml}$ of SEG solution diluted in $60 \mathrm{ml}$ of distilled water. After filtration, the PTFE membranes were placed with the SEG layer facing down towards a glass substrate covered by a thin layer of IPA. The sample was gently pressed to remove any air bubbles trapped below the PTFE membrane and placed in a hot plate at a temperature of $80^{\circ} \mathrm{C}$. After all IPA evaporates, the sample were cooled down to room temperature and the PTFE membrane was peeled off leaving a SEG film deposited on the surface. For the PDMS triboelectric layer, a previously prepared SEG coated glass sample was used as a back electrode. On top of the SEG layer, a film of polyimide was deposited to serve as a current leakage blocking layer. The PDMS solution was prepared with 10:1 proportion of polymer to catalyst and after deposition was cured for $2 \mathrm{~h}$ at $80^{\circ} \mathrm{C}$. Finally, a layer of PDMS with approximately $800 \mu \mathrm{m}$ of thickness was deposited by spin-coating, at $250 \mathrm{rpm}$ for $25 \mathrm{~s}$. In the end both counterparts were mounted with the PDMS and SEG layers facing each other with an offset resulting in an overlap of $2.5 \times 1 \mathrm{~cm}$.

Al/PDMS TENG fabrication: the PDMS triboelectric layer was made in the same way as SEG based TENG. A glass substrate covered by an aluminum foil was used as an electrode as well as an active triboelectric layer. In a similar way to the SEG based TENG, both parts were mounted with the PDMS and aluminum layers facing each other with a $2.5 \times 1 \mathrm{~cm}$ overlap.

Raman Spectroscopy: A Renishaw spectrometer was used with an excitation laser of $535 \mathrm{~nm}$ wavelength. Spectres were obtained through a 1,024-pixel detector at room temperature, with a D1 filter, a $\times 50$ objective and a spot size of approximately $2 \mu \mathrm{m}$. The analysis software used was NGSLabSpec.

Electric measurements: Three types of electric tests were performed, using a Keithley B1500A Semiconductor Device Analyzer. Sheet resistance tests were performed on SEG conductive layers after IDT transfer method. For this, a twoprobe method was applied, and I-V characteristic tests were carried out on a range from -1 to $1 \mathrm{~V}$ applied voltage. I-V time dependant tests were performed on samples with PDMS and graphene as well as on samples with PDMS, polyimide and graphene to study the attenuation of current leakage trough PDMS after applying the polyimide layer. These measurements were made with a time-based mode where the current behaviour was measured along time and a leaking value was obtained after a stabilization period. TENG's characterization was made using a time-based mode to measure both $\mathrm{V}_{\mathrm{oc}}$ and $\mathrm{I}_{\mathrm{sc}}$ output behaviour along several external load values. The applied load varied from 1 to $6 \mathrm{G} \Omega$. After output signal analysis power output and power density output were calculated using the effective contact or overlap area of the sample.

\section{RESULTS}

To evaluate the advantages of using solution processed graphene as opposed to metallic electrodes, two different types of dielectricto-conductor TENGs in a vertical separation configuration were assembled, one using SEG/PDMS and the other Al/PDMS as triboelectric pair. The individual components for these two devices (on top) and their assembly (on bottom) are 


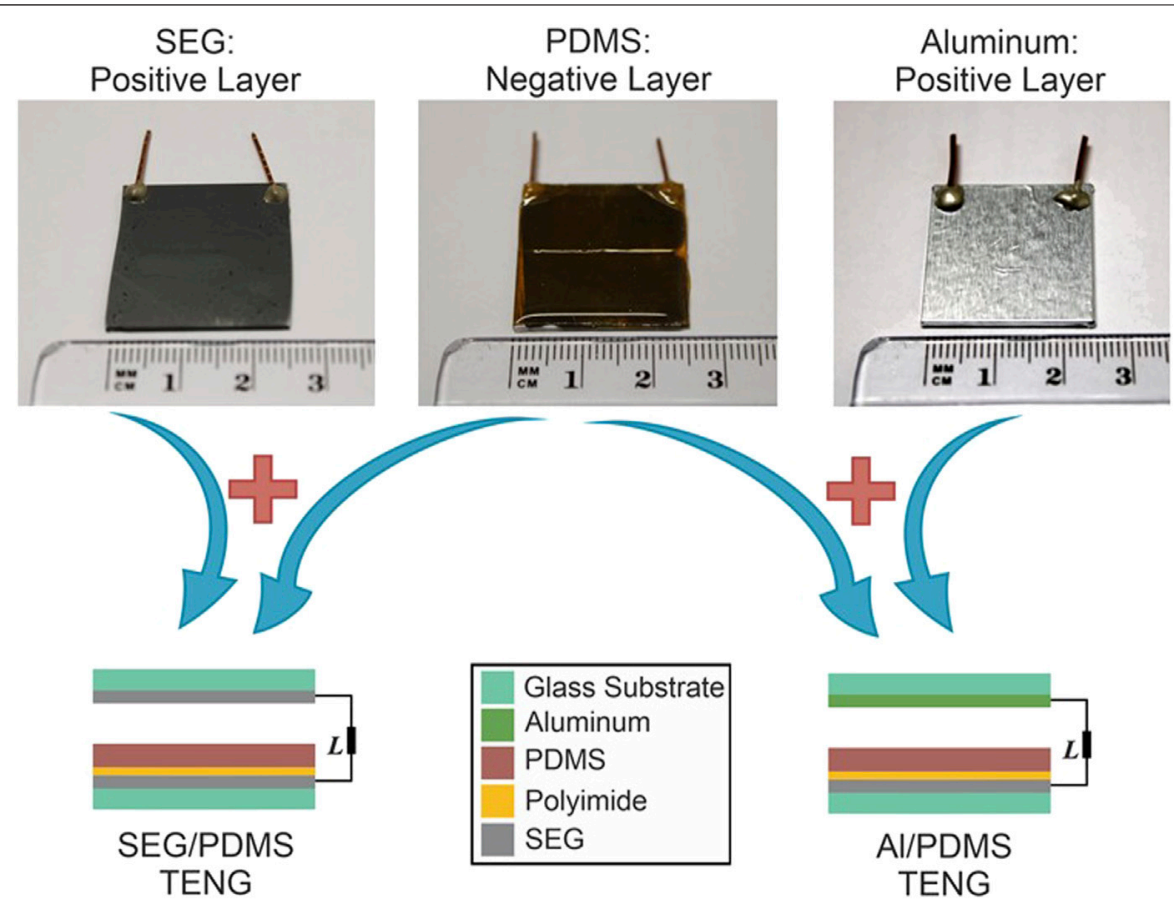

FIGURE 1 | Schematic for a SEG/PDMS TENG (bottom left) and for an Al/PDMS TENG (bottom right) with pictures of corresponding composing triboelectric parts.

schematized in Figure 1, and, as shown, both used a PDMS negative triboelectric layer deposited through a solution process with an SEG back electrode. SEG electrodes were deposited on clean glass substrates with a corona discharge surface treatment. This surface optimization was applied to enhance surface energy and promote better adhesion of SEG during the transfer process [16]. SEG films were characterized by Raman spectroscopy. Figure 2 presents the obtained Raman spectra, which shows the three characteristic peaks for graphene at $1,360 \mathrm{~cm}^{-1}$ (D), $1,570 \mathrm{~cm}^{-1}(\mathrm{G})$ and $2,730 \mathrm{~cm}^{-1}(2 \mathrm{D})$. The ratio of $\mathrm{G}$ band and $2 \mathrm{D}$ band, $\mathrm{I}_{2 \mathrm{D}} / \mathrm{I}_{\mathrm{G}}$, is approximately 0.32 and is consistent with multilayer graphene. We can also verify that we are not in the presence of graphite since the $2 \mathrm{D}$ band shows a symmetry in the peak, which should deform and arch to the right in presence of graphite. The low intensity of D band indicates low defects and impurities in the film $[17,18]$.

SEG layers present a stable and ohmic behavior with a sheet resistance of $150 \Omega / s q$, comparable to CVD graphene-based TENG, where high quality but non-solution graphene films presented $200 \Omega /$ sq [19]. In the Al/PDMS TENG, aluminum was used as the positive triboelectric layer, through lamination of an aluminum foil to glass substrate. As an alternative to aluminum, in the SEG/PDMS TENG, an additional layer of SEG acted as the positive triboelectric layer, allowing a fully solution-based process.

To evaluate potential leaking current problems through the PDMS layer time dependent electrical tests were performed. This phenomenon usually associated with an electric-field penetration depth based on the geometry of used electrodes can be affected by several aspects such as solvent entrapment, micro air bubble channels originated during deposition process or even penetration of SEG into PDMS nanopores. These tests were made using a time dependent mode with the device triboelectric layers at full permanent contact and an applied voltage of $40 \mathrm{~V}$ between electrodes. When initially assembling both TENGs the PDMS triboelectric layer was deposited directly on top of the graphene back electrode, i.e., without the polyimide layer, as shown schematically in Figure 1. When both triboelectric layers where in contact and a voltage was applied to the electrodes, a small amount of current passed through the device. Since PDMS was the only material used in these first devices that presented dielectric properties, this current indicated a leakage through this thin layer of polymeric material. To mitigate this effect, an additional layer of polyimide tape was applied on top of SEG electrode prior to PDMS deposition. This polyamide blocking layer resulted in a reduction of the mean leaking current of $82.8 \%$, from $1.45 \mathrm{pA}$ to approximately $0.25 \mathrm{pA}$ as shown in Figure 3. This strategy was used to attenuate the current leakage in both TENGs, in a structure SEG/polyimide/ PDMS which acted as the negative triboelectric part of the devices.

After attenuating the current leakage, both devices were assembled directly on a homemade set up capable of apply a linear motion with a controlled frequency ranging from 1 to $1.75 \mathrm{~Hz}$. This configuration allowed a linear separation type working mode for the devices as illustrated in Figure 4. The frequency was set to $1.5 \mathrm{~Hz}$ and the device were tested for voltage and current output for several external loads ranging from 1 to $6 \mathrm{G} \Omega$. After an initial cycling step of around $90 \mathrm{~s}$ to fully charge both triboelectric layers some electrostatic losses 


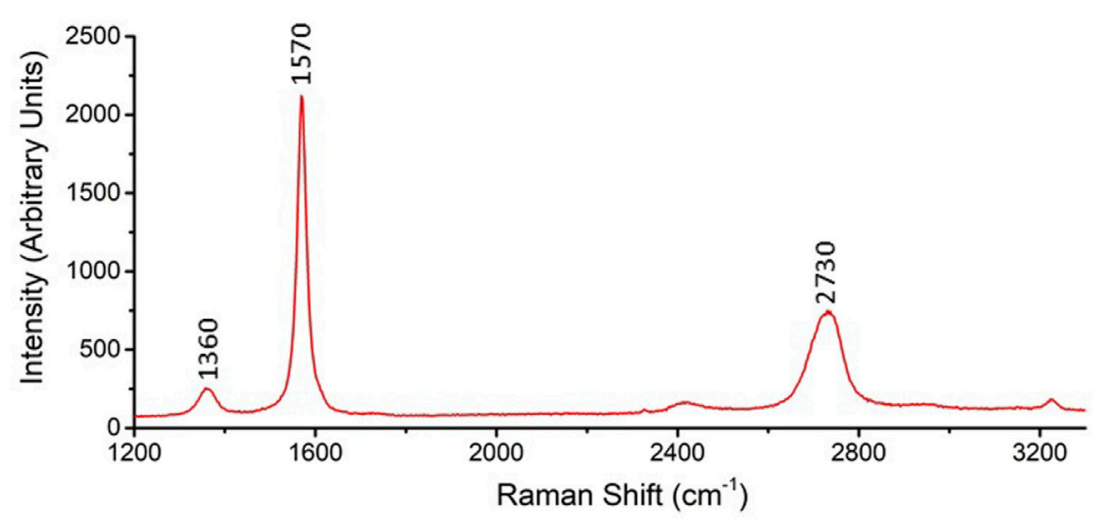

FIGURE 2 | Raman spectra for SEG films used as conductive layers in TENGs.

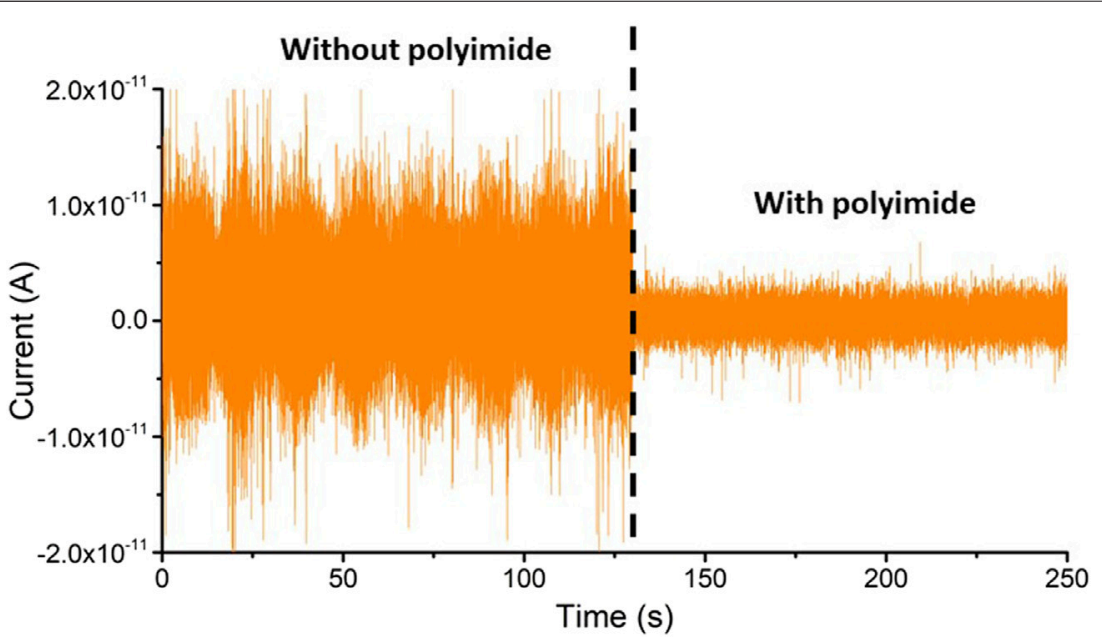

FIGURE 3 |Current leakage measurement of PDMS/SEG back electrode (left) and PDMS with a blocking layer of polyimide-PDMS/polyimide/SEG (right) with an applied voltage of $40 \mathrm{~V}$ across the device.

may occur over time. Therefore, the linear motion was always initiated approximately $5 \mathrm{~s}$ prior to the TENG's testing in order to replenish these electrostatic charges in the interface between these layers and to guarantee a stable measurement. This phenomenon was noticeable since the triboelectric potential presents an increasing output signal until it reaches a stable value.

For both TENGs, it is possible to observe an increase in voltage output with the rise of external load and a decrease in output current in this same direction as depicted in Figures 5A,C. Since the current output is driven by an electrostatic induction, it is expected that for higher external loads this induction is less effective which results in higher voltages and lower currents. This is exactly what was observed in both SEG and Al TENGs as depicted on Figure 5.

The Al/PDMS TENG presented a $\mathrm{V}_{\mathrm{oc}}$ of approximately $217 \mathrm{~V}$ and $\mathrm{I}_{\mathrm{sc}}$ of $706 \mathrm{nA}$. Voltage and current values were combined with the effective area of contact of $2.5 \times 1 \mathrm{~cm}$ to create a power density distribution according to the external load shown in Figure 5B.
This device showed a maximum power of $11.07 \mu \mathrm{W} / \mathrm{cm}^{2}$ at $600 \mathrm{M} \Omega$, which represents a $\times 40$ increase in maximum output power when compared with similar devices using Al/PDMS triboelectric pairs [20,21], revealing that the use of SEG as back-electrode instead of an aluminum is a better approach to generate higher voltage and current outputs.

In SEG-based TENG, it was possible to obtain a $\mathrm{V}_{\mathrm{oc}}$ of around $233 \mathrm{~V}$ and an I of approximately $731 \mathrm{nA}$. A maximum power density of $13.14 \mu \mathrm{W} / \mathrm{cm}^{2}$ was obtained for an external load of $200 \mathrm{M} \Omega$ at $1.5 \mathrm{~Hz}$ (Figure 5D). Compared to CVD graphene TENG when used as positive triboelectric layer with PDMS as negative layer, this reach a $\mathrm{V}_{\mathrm{oc}}$ of $18.7 \mathrm{~V}$ and Isc of $6.6 \mu \mathrm{A}$ with graphene layer uncrumpled [22]. Even though the voltage output is considerably smaller than the one obtained in this work, lower than a 10th, the current output is almost ten times higher. These discrepancies practically compensate in terms of output power, which results in a similar power density of $13 \mu \mathrm{W} / \mathrm{cm}$ [2]. These differences in current and voltage values can be related to the graphene quality since CVD grown graphene can present higher 


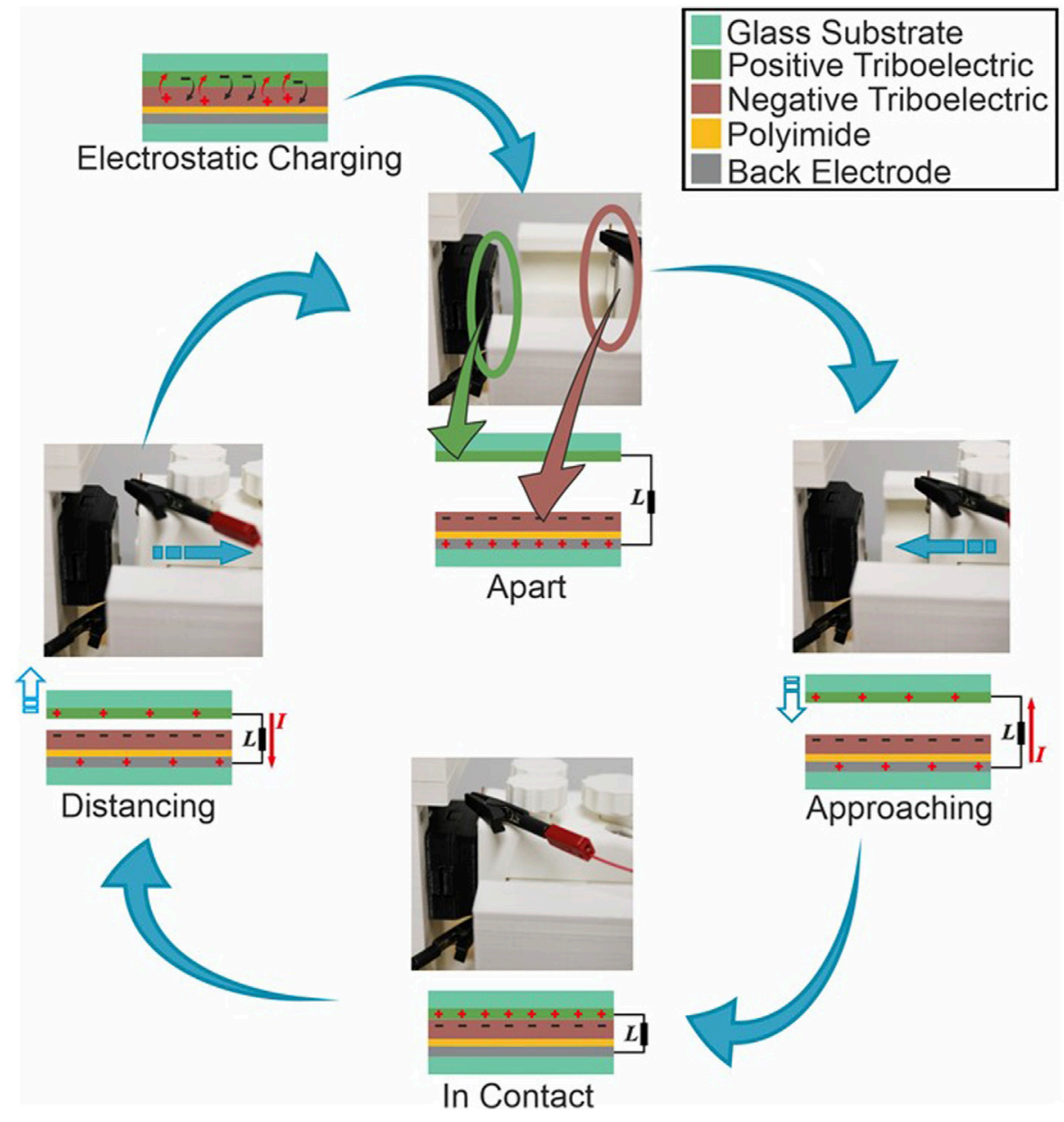

FIGURE 4 | Generic conductor-to-dielectric TENG working mechanism associated with used testing assembly.

conductivity when compared to exfoliated graphene, which can result in a better induction process and higher currents. However, this higher quality material requires more complex and expensive methods that are not scalable and will not be suitable for the textile and wearable industry.

A comparison between Al/PDMS and SEG/PDMS TENG, is shown in Figure 6, with the external load associated to the maximum power point of each device. The Al/PDMS device showed voltages around $128.54 \mathrm{~V}$ and current of $215.38 \mathrm{nA}$ at $600 \mathrm{M} \Omega$ while the SEG/PDMS reached a maximum power derived from a voltage of $80.89 \mathrm{~V}$ and a current of $406.08 \mathrm{nA}$ at $200 \mathrm{M} \Omega$. Even though SEG/PDMS presents lower voltage and higher current, with Al/PDMS showing the opposite behaviour, these small differences are negligible, and result in similar maximum power. This indicates that shear exfoliated graphene can present similar performances to aluminum films and can effectively be used to replace these metallic layers towards full solution processed devices.

A comparison of output magnitude for both devices can also be helpful to try to locate this exfoliated SEG in the triboelectric series. Based on the similar power output for both TENGs, it is possible to imply that the triboelectric difference between
PDMS and SEG is nearly the same as between PDMS and aluminum. According to these results, it is expectable this material as a close position to aluminum, close to the positive end of the triboelectric series [23] but a more detailed comparison with other different materials is needed to reach a more accurate conclusion.

\section{DISCUSSION}

With this work we demonstrated an easy, low-cost and fast process to fabricate a TENG based on the triboelectric effect of PDMS with shear exfoliated graphene. Here we fabricated and processed graphene from graphite through solution methods, which potentiate the implementation of solution-based methods and carbon-based materials using abundant and cost-effective sources. SEG thin films created in this work presented a stable and ohmic behavior with a sheet resistance comparable with values obtained in the literature for CVD graphene. With these results it was possible to support the integration of carbon-based materials into TENG devices, as alternative to the commonly used metallic layers. The potential of SEG as back-electrode is further evidenced when 

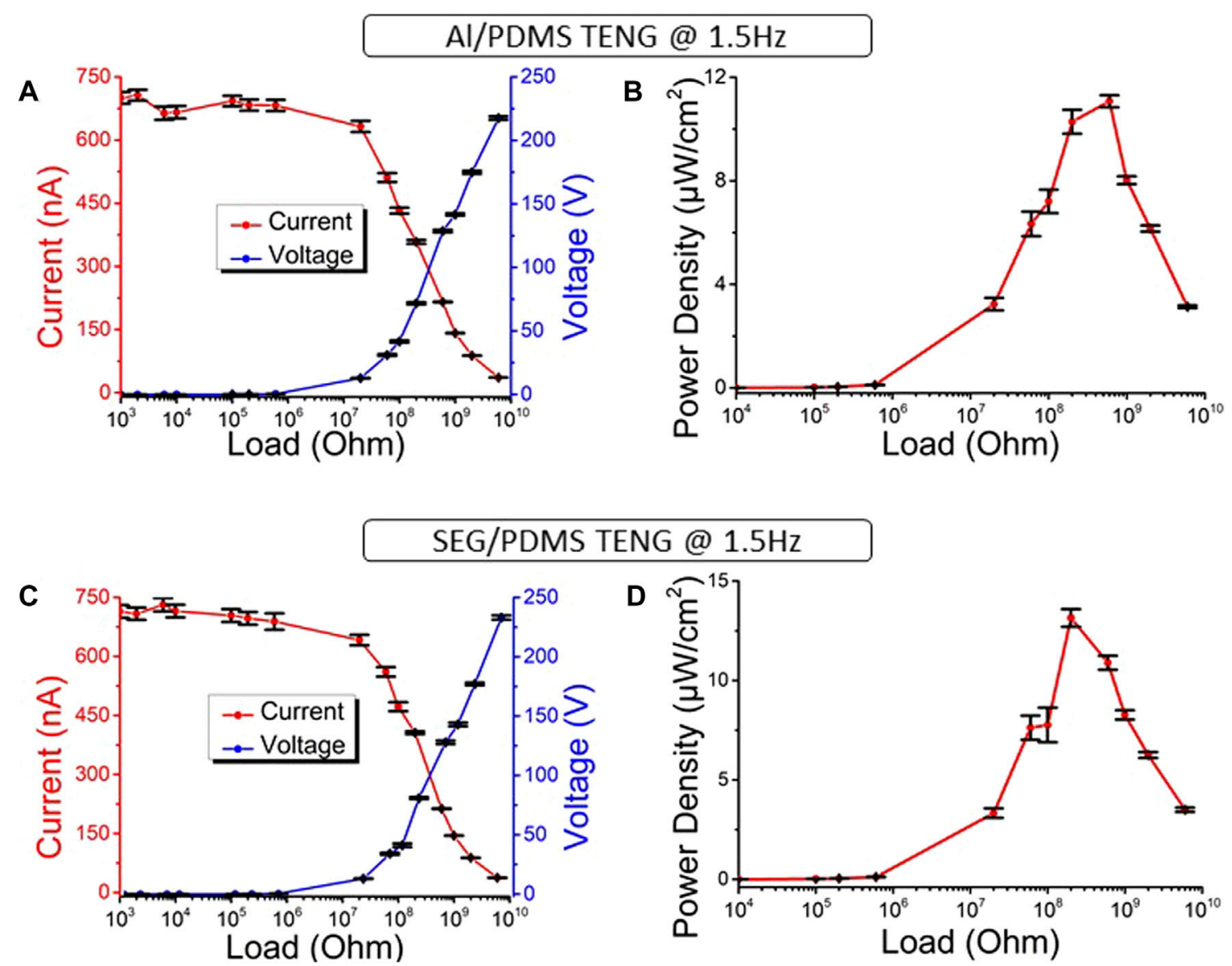

FIGURE 5 | Device output performances for several external loads, depicting voltage/current behavior and calculated power density for Al/PDMS (A,B) and SEG/ PDMS (C,D).
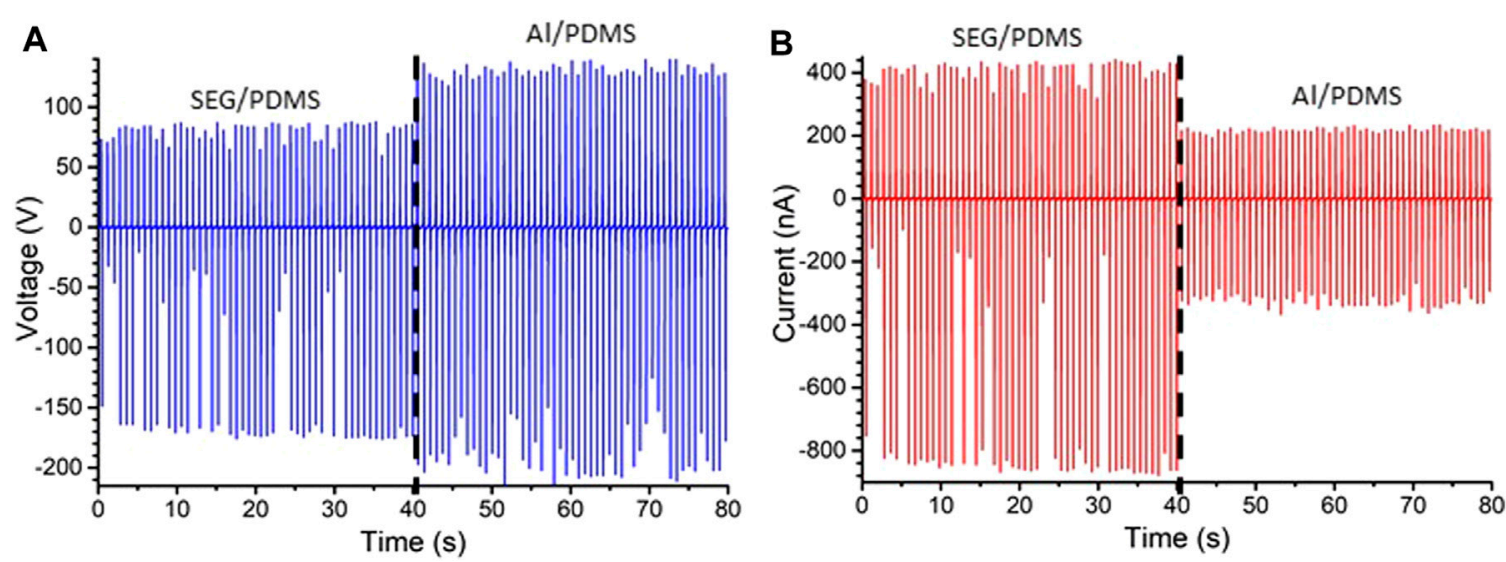

FIGURE 6 | Output values comparison for both tested devices on the calculated maximum power point of operation, showing the difference in voltage (A) and current (B) values.

compared with previous reported similar devices using aluminum as back-electrode, reaching almost 40 times higher power output values. Regarding performance of SEG as a triboelectric layer, it was possible to observe a similar performance when compared to devices using aluminum as triboelectric layer. This outcome revealed that, even though the graphene conductive layers presented higher 
sheet resistance than metallic layers, in terms of integration into triboelectric nanogenerators they can still serve as a capable substitute for conventional electrode materials as well as for triboelectric layers with the major advantage of a full solutionbased device fabrication. Therefore, these results support the integrability of carbon-based materials and demonstrate the potential of solution process for low-cost triboelectric devices. These solution-based methods allow for TENGs to be fabricated directly on end products on textile industry without substantial modification to already existing. With this it is possible to potentiate the use of graphene to produce nanogenerators and sensors aiming for health or security applications through textile and wearable devices.

\section{DATA AVAILABILITY STATEMENT}

The raw data supporting the conclusions of this article will be made available by the authors, without undue reservation.

\section{REFERENCES}

1. Prauzek M, Konecny J, Borova M, Janosova K, Hlavica J, and Musilek P. Energy Harvesting Sources, Storage Devices and System Topologies for Environmental Wireless Sensor Networks: A Review. Sensors (2018) 18(8): 2446. doi:10.3390/s18082446

2. Zhou M, Al-Furjan MSH, Zou J, and Liu W. A Review on Heat and Mechanical Energy Harvesting from Human - Principles, Prototypes and Perspectives. Renew Sustainable Energ Rev (2018) 82:3582-609. doi:10.1016/ j.rser.2017.10.102

3. Wu C, Wang AC, Ding W, Guo H, and Wang ZL. Triboelectric Nanogenerator: A Foundation of the Energy for the New Era. Adv Energ Mater. (2019) 9(1):1802906-25. doi:10.1002/aenm.201802906

4. Pu X, Hu W, and Wang ZL. Toward Wearable Self-Charging Power Systems: The Integration of Energy-Harvesting and Storage Devices. Small (2018) 14(1): 1702817-9. doi:10.1002/smll.201702817

5. Xi F, Pang Y, Li W, Jiang T, Zhang L, Guo T, et al. Universal Power Management Strategy for Triboelectric Nanogenerator. Nano Energy (2017) 37:168-76. doi:10.1016/J.NANOEN.2017.05.027

6. Zhou C, Yang Y, Sun N, Wen Z, Cheng P, Xie X, et al. Flexible Self-Charging Power Units for Portable Electronics Based on Folded Carbon Paper. Nano Res (2018) 11(8):4313-22. doi:10.1007/s12274-018-2018-8

7. Jiang C, Li X, Ying Y, and Ping J. Fluorinated Graphene-Enabled Durable Triboelectric Coating for Water Energy Harvesting. Small (2021) 17(8): 2007805-8. doi:10.1002/smll.202007805

8. Chen H, Zhang S, Zou Y, Zhang C, Zheng B, Huang C, et al. PerformanceEnhanced Flexible Triboelectric Nanogenerator Based on Gold ChlorideDoped Graphene. ACS Appl Electron Mater (2020) 2(4):1106-12. doi:10.1021/acsaelm.0c00100

9. Pace G, Ansaldo A, Serri M, Lauciello S, and Bonaccorso F. Electrode Selection Rules for Enhancing the Performance of Triboelectric Nanogenerators and the Role of Few-Layers Graphene. Nano Energy (2020) 76:104989. doi:10.1016/ j.nanoen.2020.104989

10. Pace G, Serri M, Castillo AEDR, Ansaldo A, Lauciello S, Prato M, et al. Nitrogen-Doped Graphene Based Triboelectric Nanogenerators. Nano Energy (2021) 87:106173. doi:10.1016/j.nanoen.2021.106173

11. Chu H, Jang H, Lee Y, Chae Y, and Ahn J-H. Conformal, GrapheneBased Triboelectric Nanogenerator for Self-Powered Wearable Electronics. Nano Energy (2016) 27:298-305. doi:10.1016/ j.nanoen.2016.07.009

\section{AUTHOR CONTRIBUTIONS}

AN and MC prepared SEG solution, ID fabricated the TENG microdevices and performed electrical characterization. HA conceived and supervised the project. ID and HA wrote the manuscript in collaboration with all the authors.

\section{FUNDING}

The authors acknowledge funding supported by FCT/ MCTES, co-financed by the operational program FEDER/ FNR, and by national funds, under contracts POCI-01-0145FEDER-0032072, PTDC/QUI-QIN/29834/2017, PCIF/SSO/ 0163/2019, and SFRH/BD/145261/2019. AN and MC acknowledge the support of EPSRC under awards EP/ S019855/1EP/M001024/1 and EP/M002438/1. Project CICECO-Aveiro Institute of Materials, UIDB/50011/2020 and UIDP/50011/2020.

12. Kumar A, and Joshi N. Self-Powered Environmental Monitoring Gas Sensors: Piezoelectric and Triboelectric Approaches, Nanobatteries and Nanogenerators (2021). p. 463-89. Elsevier. doi:10.1016/b978-0-12-8215487.00018-x

13. Chun S, Son W, Lee G, Kim SH, Park JW, Kim SJ, et al. Single-Layer GrapheneBased Transparent and Flexible Multifunctional Electronics for Self-Charging Power and Touch-Sensing Systems. ACS Appl Mater Inter (2019) 11(9): 9301-8. doi:10.1021/acsami.8b20143

14. Zhang W, Diao D, Sun K, Fan X, and Wang P. Study on Friction-Electrification Coupling in Sliding-Mode Triboelectric Nanogenerator. Nano Energy (2018) 48:456-63. doi:10.1016/j.nanoen.2018.04.007

15. Shin D-W, Barnes MD, Walsh K, Dimov D, Tian P, Neves AIS, et al. A New Facile Route to Flexible and Semi-transparent Electrodes Based on Water Exfoliated Graphene and Their Single-Electrode Triboelectric Nanogenerator. Adv Mater (2018) 30(39):1802953-7. doi:10.1002/adma.201802953

16. Sellin N, and Campos JSd. C. Surface Composition Analysis of PP Films Treated by Corona Discharge. Mat Res (2003) 6(2):163-6. doi:10.1590/s151614392003000200009

17. Weiss NO, Zhou H, Liao L, Liu Y, Jiang S, Huang Y, et al. Graphene: An Emerging Electronic Material. Adv Mater (2012) 24(43):5782-825. doi:10.1002/adma.201201482

18. Zhu Y, Murali S, Cai W, Li X, Suk JW, Potts JR, et al. Graphene and Graphene Oxide: Synthesis, Properties, and Applications. Adv Mater (2010) 22:3906-24. doi:10.1002/adma.201001068

19. He X, Mu X, Wen Q, Wen Z, Yang J, Hu C, et al. Flexible and Transparent Triboelectric Nanogenerator Based on High Performance Well-Ordered Porous PDMS Dielectric Film. Nano Res (2016) 9(12):3714-24. doi:10.1007/s12274-016-1242-3

20. Vivekananthan V, Kim WJ, Alluri NR, Purusothaman Y, Khandelwal G, and Kim S-J. A Highly Reliable Contact-Separation Based Triboelectric Nanogenerator for Scavenging Bio-Mechanical Energy and Self-Powered Electronics. J Mech Sci Technol (2021) 35(5):2131-9. doi:10.1007/s12206021-0429-z

21. Chandrasekhar A, Alluri NR, Saravanakumar B, Selvarajan S, and Kim S-J. A Microcrystalline Cellulose Ingrained Polydimethylsiloxane Triboelectric Nanogenerator as a Self-Powered Locomotion Detector. J Mater Chem C (2017) 5(7):1810-5. doi:10.1039/c6tc05104a

22. Chen $\mathrm{H}, \mathrm{Xu} \mathrm{Y}$, Zhang J, Wu W, and Song G. Enhanced Stretchable Graphene-Based Triboelectric Nanogenerator via Control of Surface Nanostructure. Nano Energy (2019) 58:304-11. doi:10.1016/ j.nanoen.2019.01.029 
23. He W, Fu X, Zhang D, Zhang Q, Zhuo K, Yuan Z, et al. Recent Progress of Flexible/Wearable Self-Charging Power Units Based on Triboelectric Nanogenerators. Nano Energy (2021) 84:105880. doi:10.1016/ j.nanoen.2021.105880

Conflict of Interest: The authors declare that the research was conducted in the absence of any commercial or financial relationships that could be construed as a potential conflict of interest.

Publisher's Note: All claims expressed in this article are solely those of the authors and do not necessarily represent those of their affiliated organizations, or those of the publisher, the editors and the reviewers. Any product that may be evaluated in this article, or claim that may be made by its manufacturer, is not guaranteed or endorsed by the publisher.

Copyright $\odot 2021$ Domingos, Neves, Craciun and Alves. This is an open-access article distributed under the terms of the Creative Commons Attribution License (CC $B Y)$. The use, distribution or reproduction in other forums is permitted, provided the original author(s) and the copyright owner(s) are credited and that the original publication in this journal is cited, in accordance with accepted academic practice. No use, distribution or reproduction is permitted which does not comply with these terms. 